\title{
Primary central nervous system lymphoma co-existing with ataxia-telangiectasia syndrome
}

\author{
Agnieszka Mizia-Malarz ${ }^{1 *}$ (D), Weronika Stolpa ${ }^{1}$, Magdalena Zapała $^{2}$, Bartosz Zwiernik $^{2}$, \\ Mateusz Marszałek ${ }^{1,2}$ \\ ${ }^{1}$ Department of Pediatrics, Upper Silesia Children's Healthcare Center, Medical University of Silesia, Katowice, Poland \\ ${ }^{2}$ Medical University of Silesia, Katowice, Poland
}

\section{Introduction}

Primary central nervous system lymphoma (PCNSL) is a very rarely diagnosed (<1\% cases) non-Hodgkin lymphoma (NHL) in children. The most frequently diagnosed PCNSL $(90 \%$ of cases) is diffuse large B-cell lymphoma (DLBCL) with cerebral hemisphere localization (38\% of cases) [1-4]. Signs of increased intracranial pressure and behavioral changes are the most common clinical symptoms [5]. Polychemotherapy with high-dose methotrexate is the treatment of choice. The prognosis is unsatisfactory, with 5-year survival of c.33\% $[5,6]$. In about $70 \%$ of patients with PCSNL, primary immunodeficiencies have been described [5]. One of these is ataxia-telangiectasia syndrome (ATS), which is a rare genetic form of ataxia resulting from mutation in a gene located on chromosome 11q22-23. The syndrome is a factor predisposing to the development of neoplasms, especially those derived from the lymphatic system (25\% lifetime risk) [5].

\section{Case report}

We present the case of a 2.5-year-old girl diagnosed with PCNSL. Additionally, during the diagnostic procedure ATS was diagnosed. The girl's medical history showed frequent upper respiratory tract infections, but neither she nor her family had a history of prior immunodeficiencies or cancer. For eight months, increasing neurological symptoms had been observed: dragging of the right leg while walking, right lip corner asymmetry, and speech regression. Tumor of the left cerebral hemisphere was diagnosed (Figures $1 \mathrm{~A}, \mathrm{~B})$. After the tumor's partial resection, diffuse large B-cell lymphoma (DLBCL) was diagnosed (LCA+, CD3-,
CD20-, PAX5+, MUM-1+, CD30-/+, BCL6+/-, c-MYC- (in single cells), CD10-, Tdt-, CD34-, CD99-/+, Ki-67-60\%) (assessment made by two histopathologists). After surgery, the patient's condition was moderately severe: lying position, swallowing difficulties, right nerve VII paralysis, and right hemiplegia. The child presented hormonal abnormalities (diabetes insipidus, hypothyroidism) and immunoglobulin $\mathrm{G}$ (IgG) deficiency $(320 \mathrm{mg} / \mathrm{dL})$. Follow-up magnetic resonance imaging (MRI) revealed a residual tumor. No other disease location was revealed. The girl was qualified for treatment according to Protocol Inter B NHL 2010, group C3. COP prophase was started (100\% doses) [prednisone per os (p.o.), vincristine (i.v.), cyclophosphamide i.v., methotrexate intrathecal (i.t.), hydrocortisonum i.t., cytarabine i.t.]. After this course, the patient's condition worsened. Head computed tomography revealed a large hydrocele over the left cerebral hemisphere (Figures 1C, D). A ventriculoperitoneal shunt valve system was implanted. Due to the challenging clinical condition of the child, the scheduled course of chemotherapy was abandoned, and was replaced with waiting R-COP (100\% doses) (rituximab i.v. +COP). Deterioration of the clinical condition was observed: periodic respiratory disorders, cardiac arrhythmias, and a permanent tendency to hypernatremia. She was discharged from our department upon her parents' request in a moderate condition, with the purpose of continued treatment abroad. After 11 days, she was admitted to the Charité University Hospital in Berlin, Germany.

The diagnostics was extended to include genetic testing. Ataxia-telangiectasia syndrome was diagnosed based on the presence of two pathogenic mutations in ATM gene located on chromosome 11 q22;23 (double heterozygote).

\footnotetext{
*Address for correspondence: Agnieszka Mizia-Malarz, Department of Pediatrics, Upper Silesia Children's Healthcare Center, Medical University of Silesia, Medykow 16, 40-752 Katowice, Poland, e-mail: amizia-malarz@sum.edu.pl
}

\section{PTHiT Copyright $\odot 2021$}

The Polish Society of Haematologists and Transfusiologists, Insitute of Haematology and Transfusion Medicine. All rights reserved. 


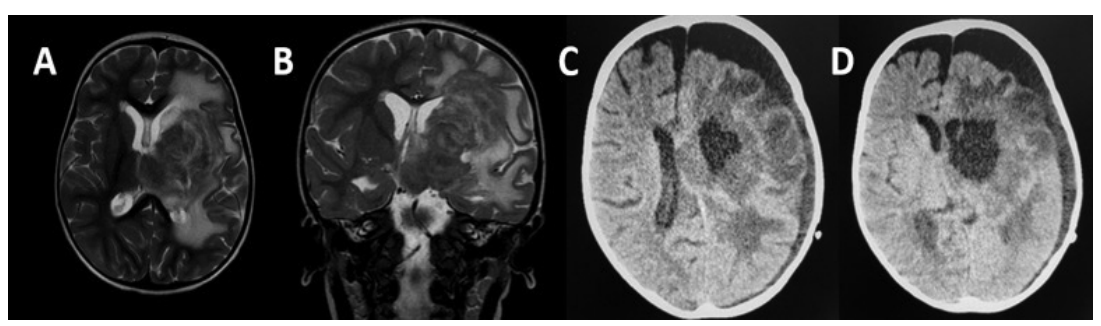

Figures 1. Head magnetic resonance imaging (MRI) before neurosurgical resection of primary central nervous system lymphoma: A. Axial T2-weighted MRI; B. Coronal T2-weighted MRI. Deep structures of left cerebral hemisphere show a polycyclic, non-uniform tumor of $40 \times 45 \times 68 \mathrm{~mm}(\mathrm{TR} \times \mathrm{AP} \times \mathrm{CC})$. Additional mass effect in form of shifting of central brain structures to right. In left hemisphere, in left cerebral peduncle, in pons, in medial section of upper cerebellar peduncle on right, and round left wall of $4^{\text {th }}$ ventricle, an area of white matter edema is present. C, D. Head computed tomography performed on day 5 of treatment according to Protocol Inter NHL $2010-$ large hydrocele is seen over left cerebral hemisphere, with increased mass effect and shifting of medial structures to right

Testing of the parents revealed that they were carriers of single mutant ATM alleles.

Chemotherapy according to Protocol NHL-BFM was applied (50\% doses) (dexamethasone, methotrexate, vincristine, ifosfamide, cytarabine, etoposide). Additionally rituximab (100\% dose) was given. Treatment was complicated by a systemic infection (Pseudomonas aeruginosa). This girl died 105 days after diagnosis as a result of cardiovascular insufficiency.

\section{Discussion}

PCNSL is a very rare diagnosis. A retrospective analysis of the years 1978-2008 conducted by The International Primary CSN Lymphoma Collaborative Group (IPCG) revealed only 29 pediatric cases of PCNSL [1]. Attarbaschi et al. [3], in their analysis of 1991-2019, found 75 cases of PCNSL among children and adolescents. In another study conducted by Kai et al. [2] over 20 years, only $1.5 \%$ of PCNSL cases involved children.

Patients with PCNSL often show primary immunodeficiency syndromes. In the study by Attarbaschi et al. [3], 14/75 (19\%) patients with PCNSL had concurrent immunity disorders.

In an extensive analysis of 213 cases of non-Hodgkin lymphomas (including PCNSL) in a population of children and adolescents, 124 patients revealed an increased risk of co-existing DNA repair defects and primary immunodeficiency. The largest group (32/124 patients, 26\%) comprised subjects with ATS [6].

An analysis conducted by Kim et al. [7] concerning expression of ATM protein mutation in patients with PCNSL-DLBCL (42 patients) and non-CNS-DLBCL (33 patients) showed statistically significantly more frequent $(p<0.001)$ mutation in ATM gene in patients with PCNSL-DLBCL (21/42 patients, 50\%) compared to patients with non-CNS-DLBCL (0/33 patients). The authors concluded from the study that ATM protein mutation predisposes especially to the development of proliferative diseases of the lymphatic system in the central nervous system [8].

That is why a thorough analysis of molecular defects and related immunity disorders may give hope for a better and more effective therapy in the context of an increased risk of cancer [9].

The presented case confirms the necessity of diagnostics in children with PCNSL for congenital immunodeficiency, as well as follow-up of patients with immunity deficits in order to detect cancer early enough in this group. Because of the lack of satisfactory treatment for such rare cases, its publication is very important.

\section{Acknowledgments}

The authors thank Dr Anne Thorwarth from the Department of Pediatric Hematology and Oncology, Charité - Universitätsmedizin Berlin, Germany, for information about the results of our patient.

\section{Authors' contributions}

AMM work concept; all authors manuscript writing, literature collection, critical revision and approval.

\section{Conflict of interest}

None.

\section{Financial support \\ None.}

\section{Ethics}

The work described in this article has been carried out in accordance with The Code of Ethics of the World Medical Association (Declaration of Helsinki) for experiments involving humans; EU Directive 2010/63/EU for animal experiments; Uniform requirements for manuscripts submitted to biomedical journals. 


\section{References}

1. Abla $\mathrm{O}$, Weitzman S, Blay JY, et al. Primary CNS lymphoma in children and adolescents: a descriptive analysis from the International Primary CNS Lymphoma Collaborative Group (IPCG). Clin Cancer Res. 2011; 17(2): 346-352, doi: 10.1158/1078-0432.CCR-10-1161, indexed in Pubmed: 21224370.

2. Kai Y, Kuratsu J, Ushio Y. Primary malignant lymphoma of the brain in childhood. Neurol Med Chir (Tokyo). 1998; 38(4): 232-237, doi: 10.2176/nmc.38.232, indexed in Pubmed: 9631639.

3. Attarbaschi A, Abla O, Ronceray L, et al. Primary central nervous system lymphoma: initial features, outcome, and late effects in 75 children and adolescents. Blood Adv. 2019; 3(24): 4291-4297, doi: 10.1182/bloodadvances.2019001062, indexed in Pubmed: 31869415.

4. Küker W, Nägele T, Korfel A, et al. Primary central nervous system lymphomas (PCNSL): MRI features at presentation in 100 patients. J Neurooncol. 2005; 72(2): 169-177, doi: 10.1007/s11060-0043390-7, indexed in Pubmed: 15925998.

5. Han $\mathrm{CH}$, Bathelor TT. Diagnosis and management of primary central nervous system lymphoma. Cancer. 2017; 123(22): 4314-4324, doi: 10.1002/cncr.30965, indexed in Pubmed: 28950405.
6. Giulino-Roth L, Abla O, Batchelor TT. Management of primary central nervous system lymphoma in children. Hematology Am Soc Hematol Educ Program. 2016; 2016(1): 386-389, doi: 10.1182/asheducation-2016.1.386, indexed in Pubmed: 27913505.

7. Kim SH, Cheong JW, Park KH, et al. Comparison of ataxia-telangiectasia mutated protein expression in diffuse large B-cell lymphomas of primary central nervous system and non-central nervous system origin. Arch Pathol Lab Med. 2007; 131(3): 457-467, doi: 10.5858/2007-131-457-COAMPE, indexed in Pubmed: 17516749.

8. Rothblum-Oviatt C, Wright J, Lefton-Greif MA, et al. Ataxia telangiectasia: a review. Orphanet J Rare Dis. 2016; 11(1): 159, doi: 10.1186/ s13023-016-0543-7, indexed in Pubmed: 27884168.

9. Attarbaschi A, Carraro E, Abla O, et al. European Intergroup for Childhood Non-Hodgkin Lymphoma (EICNHL) and the International Berlin-Frankfur t-Münster (i-BFM) Study Group. Non-Hodgkin lymphoma and pre-existing conditions: spectrum, clinical characteristics and outcome in 213 children and adolescents. Haematologica. 2016; 101(12): 1581-1591, doi: 10.3324/haematol.2016.147116, indexed in Pubmed: 27515251.

10. Verhoeven D, Stoppelenburg AJ, Meyer-Wentrup F, et al. Increased risk of hematologic malignancies in primary immunodeficiency disorders: opportunities for immunotherapy. Clin Immunol. 2018; 190: 22-31, doi: 10.1016/j.clim.2018.02.007, indexed in Pubmed: 29499421. 